\title{
Migraciones y diferenciación sociodemográfica del espacio residencial urbano. El caso de la ciudad de Viña del Mar en su contexto metropolitano*
}

Migrations and sociodemographical differentiation of the urban residential space. The case of the city of Viña del Mar in its metropolitan context

\author{
Carlos Valdebenito \\ Historial del artículo \\ Recibido: \\ 14 de octubre 2019. \\ Aceptado: \\ 8 de noviembre de 2019. \\ a Pontificia Universidad Católica de Valparaíso,Valparaíso, Chile. Correo electrónico: carlos.valdebenito@pucv.cl \\ * Este artículo difunde resultados de una investigación en curso que ha contado con el apoyo financiero de CONICYT, a \\ través del proyecto FONDECYT 11160747 "Ni tan cerca ni tan lejos. Transformaciones urbanas y diferenciación del espacio \\ residencial en el área metropolitana de Valparaíso, 1992-217".
}

\section{Palabras clave}

Análisis multivariante, mercado inmobiliario, migraciones interiores, migraciones internacionales

\section{Keywords}

Internal migration, international migration, multivariate analysis, real estate market

\section{Resumen}

La ciudad de Viña del Mar, desde sus orígenes hasta nuestros días, ha sido objeto de movimientos migratorios significativos, tanto en términos de su volumen como de su perfil sociodemográfico. El artículo describe, analiza e interpreta la huella de este fenómeno sociodemográfico, en la estructura del espacio residencial de la ciudad, en los últimos veinticinco años, período donde recupera fuerza y vigor. El estudio se inscribe en el campo de la ecología factorial, implementando un método estadístico de análisis multivariante a matrices de datos construidas a partir del procesamiento de las bases de los censos de población y de viviendas de 1992, 2002 y 2017. La unidad de análisis es la zona censal, unidad espacial más desagregada disponible para este tipo de estudio en el último censo levantado en Chile. Los resultados obtenidos evidencian que la distribución residencial de la migración en la ciudad, tanto interior como internacional, consolida en el período un patrón socioeconómico de estructuración del espacio residencial, como correlato de transformaciones en la estructura sociodemográfica de la población y de las actuaciones del mercado inmobiliario habitacional.

\section{Abstract}

The city of Viña del Mar, from its origins to the present day, has been subject to significant population migratory movements, both in terms of its volume and its sociodemographic profile. This article describes, analyzes and interprets the footprint of this sociodemographic phenomenon, looking at the structure of the residential space of the city, in the last twenty-five years, a period where it regains strength and vigor. The study falls within the field of factorial ecology, implementing a statistical method of multivariate analysis of data matrices constructed from the processing of the population and housing census bases of 1992, 2002 and 2017. The unit of analysis is the census zone, the most disaggregated space unit available for this type of study in the last census taken in Chile. The results obtained show that the residential distribution of migration towards the city, both within the country and from abroad, strengthen in the period a socio-economic pattern of residential space-structuring, as a correlation of transformations in the sociodemographic structure of the population and the actions of the housing real estate market. 


\section{Introducción}

El fenómeno de las migraciones interurbanas e internacionales y su impacto, tanto en el sistema de ciudades, en la estructura socioresidencial de estas, como en las condiciones de existencia de quienes lo protagonizan, ha sido de preocupación sistemática en el campo de las ciencias sociales en general y de la Geografía, en particular. A esta preocupación global, que se concentra en los territorios de origen y, con particular énfasis, en los de destino de los procesos migratorios, Chile se integra de manera sostenida hace poco más de dos décadas (Contreras, Ala-Louko, \& Labbé, 2015; Escolano, Ortiz \& Moreno, 2018; Ortiz \& Escolano, 2013; Ortiz \& Morales, 2002; Ortiz \& Schiappacasse, 1997, Ortiz \& Escolano, 2013).

El incremento significativo de las migraciones internacionales acaecidas en los últimos años en Chile (Instituto Nacional de Estadísticas y Departamento de Extranjería y Migración, 2019), aún con las proyecciones oficiales disponibles que estiman una estabilización a la baja de este fenómeno en la próxima década, que considera la puesta en régimen de nuevas reglas migratorias (INE, 2018), fundamenta la pertinencia de examinar el efecto de este fenómeno, interrelacionado con otras variables socioeconómicas y demográficas, en la estructura socioresidencial de ciudades, metropolitanas e intermedias, de destino.

En esta misma línea argumental, se inscriben los resultados recientemente publicados de un estudio que examinó la dinámica y estructura de las migraciones interurbanas en Chile (Escolano et al., 2018), referido al período 1987-2002, que informan de la pertinencia de examinar este tipo de migraciones residenciales en las áreas urbanas del país, en general, y en las ciudades de más de 50.000 residentes, en particular, y su impacto en la mantención, profundización o superación de las diferencias socioresidenciales que se observan en este tipo de ciudades en Latinoamérica en general y Chile en particular (Contreras, 2015; Contreras, 2017; Escolano et al., 2018; García-Almirall et al., 2008= ; Gil, Bayona \& Pujadas, 2013; Gil, Bayona \& Pujadas, 2015; González \& Villeneuve, 2006; Janoschka, 2016; Marcos \& Mera, 2018; Matossian, 2010; Matossian, 2015; Ortiz \& Escolano, 2013; Pujadas, 2009; Valdebenito, 2014, Janoschka, 2016).
Viña del Mar, núcleo del área urbano funcional, es una de las ciudades de Chile que, en las últimas tres décadas, experimenta significativas transformaciones en los campos socioeconómico, urbanístico y demográfico, que fueron recogidas por las estadísticas oficiales ${ }^{1}$, particularizándola en el contexto de su área metropolitana, de la región de Valparaíso y del país. Entre estas, las migraciones, internas e internacionales, tanto de corta como de larga trayectoria, dada su configuración sociodemográfica, es una de las más significativas (Valdebenito, 2014).

Distinguir y describir el efecto de la movilidad residencial, intercomunal e internacional, que tiene como destino la ciudad de Viña del Mar en el contexto del área metropolitana que conforma, en la diferenciación social de su espacio residencial, es la preocupación en la que se inscriben los resultados de la investigación en curso que se presentan en esta comunicación. El focalizar este ámbito de preocupación en Viña del Mar (Figura 1), la sexta en tamaño poblacional y la cuarta en stock de viviendas particulares de Chile (INE, 2018), se fundamenta en el volumen y en la particularidad del perfil sociodemográfico de quienes protagonizan este fenómeno demográfico.

La ciudad de Viña del Mar, con sus aproximadamente 335 mil residentes registrados en el último censo de 2017, concentra aproximadamente el $34 \%$ de la población del área metropolitana del Gran Valparaíso ${ }^{2}$. Complementariamente, sus inmigrantes de larga ${ }^{3} \mathrm{y}$ de reciente ${ }^{4}$ trayectoria, intercomunales e internacionales, representan el 35\% y el $36 \%$ del total de cada uno de estos tipos de migrantes residentes en esta área urbana funcional, respectivamente. Estos resultados informan que la ciudad ha mantenido su liderazgo en términos de tamaño poblacional y de población inmigrante, registrado desde 1992 (Tablas 1 y 2).

La investigación se inscribe en aquella línea que se orienta a develar los principios estructurantes de la diferenciación socioresidencial que se registra en las ciudades contemporáneas, tanto en Europa como en Estados Unidos y Latinoamérica (Duhau, 2013; Florida, 2017; Janoschka, 2016; Leal \& Sorando, 2016; Musterd, Marcińczak, Van Ham, \& Tammaru, 2017; Nel-lo, 2015; Tammaru, Van Ham, Marcińczak \& Musterd, 2015;

\footnotetext{
${ }^{1}$ Se hace referencia a las estadísticas sociodemográficas, a las estadísticas socioeconómicas y a las urbanísticas producidas por el Ministerio de Desarrollo Social, INE, Ministerio de Vivienda y Urbanismo, Serviu y la Dirección de Obras Municipales, cuya unidad de análisis espacial más desagregada, en general, es la comuna.

${ }^{2}$ El Gran Valparaíso como área urbano funcional de tipo metropolitano, de acuerdo con la metodología de la OCDE (2013), está conformado por las comunas de Valparaíso, Viña del Mar, Concón, Quilpué, Villa Alemana y Limache.

${ }^{3}$ Se refiere a la población que nació en otra comuna o país.

${ }^{4}$ Se refiere a la población que hace cinco años atrás (2012 en el caso del censo de 2017), residía en otra comuna o país.
} 

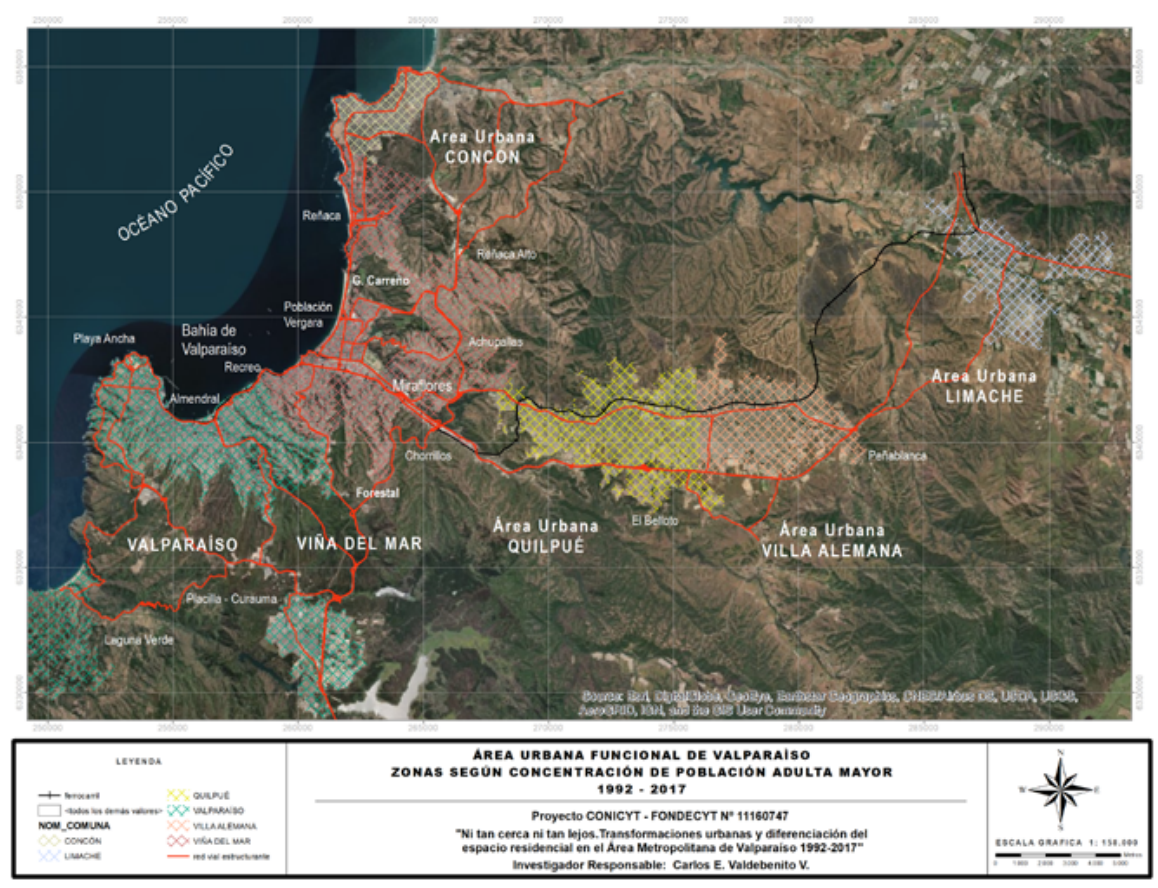

Figura 1. Viña del Mar en el contexto del área urbano funcional del Gran Valparaíso. Fuente: Elaboración propia.

Tabla 1

Distribución de los migrantes de larga trayectoria en el Gran Valparaíso.

\begin{tabular}{l|ccc|ccc}
\hline \multirow{2}{*}{ Nombre comuna } & \multicolumn{5}{|c}{ Población nacida en otra comuna o país } \\
\cline { 2 - 7 } & \multicolumn{7}{|c}{ Número } & \multicolumn{3}{c}{ Porcentaje en la Ciudad } \\
\hline \multirow{3}{*}{ Valparaíso } & $\mathbf{1 9 9 2}$ & $\mathbf{2 0 0 2}$ & $\mathbf{2 0 1 7}$ & $\mathbf{1 9 9 2}$ & $\mathbf{2 0 0 2}$ & $\mathbf{2 0 1 7}$ \\
Viña del Mar & 75.745 & 77.799 & 97.647 & 26,78 & 28,19 & 32,92 \\
Concón & 141.210 & 142.822 & 170.579 & 49,47 & 49,78 & 51,03 \\
Quilpué & 7.979 & 21.189 & 27.680 & 42,58 & 65,66 & 65,67 \\
Villa Alemana & 62.044 & 78.514 & 92.719 & 59,54 & 61,06 & 61,12 \\
Limache & 47.410 & 63.404 & 82.584 & 66,15 & 66,31 & 65,26 \\
Área Metropolitana & 14.487 & 16.935 & 20.314 & 41,44 & 43,18 & 44,05 \\
\end{tabular}

Fuente: Elaboración propia.

Tabla 2

Distribución de los migrantes recientes en el Gran Valparaíso.

\begin{tabular}{l|ccc|ccc}
\hline \multirow{2}{*}{ Nombre comuna } & \multicolumn{5}{|c}{ Migración interior e internacional reciente } \\
\cline { 2 - 7 } & \multicolumn{7}{|c}{ Número } & \multicolumn{3}{c}{ Porcentaje en la ciudad } \\
\hline & 1992 & $\mathbf{2 0 0 2}$ & $\mathbf{2 0 1 7}$ & $\mathbf{1 9 9 2}$ & $\mathbf{2 0 0 2}$ & $\mathbf{2 0 1 7}$ \\
Valparaíso & 29.188 & 30.083 & 45.822 & 10,32 & 10,90 & 15,45 \\
Viña del Mar & 46.002 & 43.627 & 66.445 & 16,11 & 15,20 & 19,88 \\
Concón & 2.572 & 8.751 & 10.715 & 13,73 & 27,12 & 25,42 \\
Quilpué & 18.532 & 26.754 & 28.169 & 17,78 & 20,81 & 18,57 \\
Villa Alemana & 14.741 & 21.908 & 25.838 & 20,57 & 22,91 & 20,42 \\
Limache & 4.326 & 5.398 & 7.447 & 12,37 & 13,76 & 16,15 \\
Área Metropolitana & 115.361 & 136.521 & 184.436 & 14,46 & 15,90 & 18,49
\end{tabular}

Fuente: Elaboración propia. 
Tabla 3

Esquema del Modelo de Áreas Sociales.

\begin{tabular}{|c|c|c|c|c|c|c|}
\hline $\begin{array}{l}\text { Postulados } \\
\text { de la sociedad } \\
\text { industrial }\end{array}$ & $\begin{array}{l}\text { Estadísticas de las } \\
\text { tendencias sociales }\end{array}$ & $\begin{array}{l}\text { Cambios en la estructura } \\
\text { de una sociedad }\end{array}$ & $\begin{array}{l}\text { Construcciones } \\
\text { analíticas, } \\
\text { dimensiones o } \\
\text { factores }\end{array}$ & $\begin{array}{l}\text { Sistema de medición } \\
\text { de las categorías } \\
\text { (índices) }\end{array}$ & & \\
\hline $\begin{array}{l}\text { Cambios en la gama } \\
\text { e intensidad de } \\
\text { relaciones }\end{array}$ & $\begin{array}{l}\text { Transformación en } \\
\text { la distribución de las } \\
\text { tareas }\end{array}$ & $\begin{array}{l}\text { Cambios en el abanico de } \\
\text { ocupaciones basados en la } \\
\text { producción }\end{array}$ & Rango social & $\begin{array}{l}\text { Ocupación, grado } \\
\text { escolar, alquiler. }\end{array}$ & इ흠 & \\
\hline $\begin{array}{l}\text { Diferenciación de } \\
\text { funciones }\end{array}$ & $\begin{array}{l}\text { Transformación de la } \\
\text { estructura productiva }\end{array}$ & $\begin{array}{l}\text { Cambios en los modos de } \\
\text { vida: incorporación de la } \\
\text { mujer en el trabajo }\end{array}$ & Urbanización & $\begin{array}{l}\text { Fecundidad, mujeres } \\
\text { activas, unidades de } \\
\text { alojamiento unifamiliares }\end{array}$ & $\begin{array}{l}\bar{\vdots} \\
\text { 햏 } \\
\stackrel{0}{=}\end{array}$ & 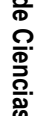 \\
\hline $\begin{array}{l}\text { Complejidad en la } \\
\text { organización }\end{array}$ & $\begin{array}{l}\text { Movilidad creciente } \\
\text { entre la población }\end{array}$ & $\begin{array}{l}\text { Redistribución espacial: } \\
\text { aislamiento y segregación } \\
\text { de grupos }\end{array}$ & Segregación & $\begin{array}{l}\text { Grupos raciales y } \\
\text { nacionales. }\end{array}$ & $\begin{array}{l}\bar{z} \\
\overline{\bar{o}} \\
\stackrel{0}{\equiv}\end{array}$ & $\frac{\infty}{\Phi}$ \\
\hline
\end{tabular}

Fuente: Rodríguez-Jaume, 2000.

Valdebenito, 2014; Zambon, Serra, Sauri, Carlucci \& Salvati, 2017). Una línea que encuentra sus raíces en la Ecología Urbana cultivada en la Escuela de Sociología de la Universidad de Chicago.

En Latinoamérica, el aterrizaje tardío de este enfoque teórico-metodológico (González \& Villeneuve, 2006) conlleva que solo a partir de la década de los ochenta del siglo XX se emprendan investigaciones en torno a la estructura socioresidencial de algunas ciudades, principalmente metropolitanas, con protocolos metodológicos multivariados (\& Buzai, 2003;Natera y Gómez, 2007 ; Ortiz \& Schiappacasse, 1998; Valdebenito, 2014). Gran parte de estas investigaciones comparten como referente teórico el modelo de áreas sociales de Shevky \& Bell (Tabla 3), aunque también emergen investigaciones que, complementaria o alternativamente, incorporan nuevas dimensiones y variables a las establecidas en el modelo original (Checa \& Arjona, 2006).

En las investigaciones realizadas en las últimas dos décadas, cuatro es el número de componentes que más prevalecen en las soluciones factoriales que explicarían la estructura socioresidencial de las ciudades metropolitanas en general y latinoamericanas en particular. Son ocho los componentes que más prevalecen en las soluciones factoriales en comento, a saber: i. Estratificación socioeconómica; ii. Estructura demográfica; iii. Estructura de los hogares; iv. Movilidad residencial; v. Estructura ocupacional; vi. Nivel educativo; vii. Saneamiento y confort ambiental y viii. Composición del parque habitacional. De acuerdo a la literatura especializada, la mayor o menor complejidad de una urbe puede ser sugerida por el número de factores asociados a la estructura de su espacio socioresidencial (González \& Villeneuve, 2006).

El artículo se organiza en tres acápites, de desarrollo progresivo y acumulativo, en el primero, se describe las principales decisiones en el protocolo multivariado implementado; el segundo acápite pone énfasis en los resultados registrados en la ciudad, en el contexto del área metropolitana del Gran Valparaíso y, finalmente, en el acápite tres, se comparte una reflexión en relación a la significación de estos movimientos migratorios en la superación o profundización de la diferenciación social del espacio residencial.

\section{Materiales y métodos}

El estudio se inscribe en el campo de la ecología factorial, lo que involucró el uso de un método estadístico de análisis multivariante aplicado a matrices de datos construidas a partir del procesamiento de las bases de los censos de población y de viviendas de 1992, 2002 y 2017. La unidad de análisis utilizada es la zona censal ${ }^{5}$.

${ }^{5}$ Se adopta como respuesta a la decisión del Instituto Nacional de Estadísticas de Chile, INE, en orden a definirla como la unidad espacial más desagregada para disponer de la base de datos del censo abreviado de población y de viviendas de 2017. 
Las decisiones acerca de la fuente de datos y de la unidad de análisis espacial implicaron costos para la investigación, imposibles de resarcir utilizando otra alternativa, a saber: i. Dado el carácter abreviado del censo implementado el año 2017, lo que implicó dejar de interrogar en relación a significativas variables socioeconómicas, como el tipo de ocupación, se requirió utilizar indicadores alternativos a los tradicionalmente usados para aproximarnos a la estratificación socioeconómica de los hogares. ii. La unidad espacial más desagregada disponible para procesar los datos del censo de población de 2017 es la zona censal, lo que impidió construir, como lo habíamos hecho en una investigación anterior para los años 1992 y 2002 (Valdebenito, 2014), unidades espaciales geográficamente más significativas, unidades vecinales y sectores en el caso de Viña del Mar.

Luego de un análisis exploratorio, se construyeron tres matrices de datos de veintisiete indicadores cada una, organizados en torno a seis variables, correspondientes a los años 1992, 2002 y 2017, que, además de cumplir las exigencias estadísticas del análisis factorial, informaban apropiadamente de los procesos de transformación socioeconómica, demográfica y del parque de viviendas particulares acaecida en el período 1992-2017, así como de los procesos migratorios, interiores e internacionales de larga y corta trayectoria, objeto de preocupación de esta comunicación.

Las variables e indicadores examinadas a través del análisis factorial de componentes principales, se detallan a continuación: 1. Estratificación socioeducativo ${ }^{6}$ : i. Hogares nivel bajo (v1); ii. Hogares nivel medio-bajo (v2); iii. Hogares nivel medio-alto (v3); iv. Hogares nivel alto (v4); v. Jefes de hogar con estudios de posgrado (v5) y vi. Promedio de años de estudio de la jefatura de hogar (v10); 2. Situación laboral: i. Hombres activos económicamente (v6); ii. Hombres inactivos (v7); iii. Mujeres activas económicamente (v8); iv. Mujeres inactivas (v9); 3. Estructura demográfica: i. Índice de longevidad (v11); ii. Índice de juventud ${ }^{8}$ (v12); iii. Índice de vejez (v13); 4. Tipos de Hogar: i. Hogar unipersonal (v14); ii. Hogar nuclear (v15); iii. Hogar extendido (v16); iv. Hogar compuesto (v17); v. Hogar sin núcleo (v18); 5. Movilidad residencial: i. Población que hace cinco años residía en otra comuna (v19); ii. Población que hace cinco años residía en otro país (v20); iii. Población que nació en otra comuna (v21); iv. Población que nació en otro país (v22); 6. Tipos de vivienda particular: i. Vivienda tipo casa (v23); ii. Vivienda tipo departamento en edificio (v24); iii. Vivienda tipo no consolidada (v25); iv. Vivienda calidad global irrecuperable (v26); v. Viviendas particulares desocupadas (v27).

Al ser construidas las matrices, a través del software IBMSPSS 24, se procedió al análisis factorial. Las decisiones más significativas adoptadas en este proceso metodológico se describen a continuación como una forma de iluminar el examen y valoración de los resultados de la investigación que se presentan en este artículo ${ }^{10}$.

Las exigencias, tanto teóricas como estadísticas, que busca resguardar el análisis exploratorio básicamente dicen relación con distribución normal y correlación. No existe consenso en relación a la pertinencia de la exigencia de normalidad, en estudios que trabajan con el universo poblacional ${ }^{11}$, como el caso de este estudio. Sin desmedro de lo dicho, se tomó la decisión de normalizar la matriz de datos previo a examinar su correlación.

Los test de linealidad aplicados, que acreditan que las matrices del estudio se ajustan a un modelo de análisis

\footnotetext{
${ }^{6} \mathrm{Al}$ no disponer de datos referidos ni los ingresos ni a la ocupación de las jefaturas de hogar, se optó por una aproximación a la estratificación socioeconómica que examina exclusivamente la variable nivel de estudios. Si se sigue lo consignado por Molinatti (2013), "para evitar el efecto distorsionador de la estructura etaria sobre la educación de una población y procurando captar a un grupo de particular influencia se trabajó solo con los jefes y las jefas de hogar de 30 a 59 años". Se distingue como un hogar de estrato alto, aquel cuya jefatura tiene estudios superiores completos, tanto de pre como postgrado. Un hogar de estrato medio-alto es aquel cuya jefatura tiene estudios medios completos y superiores incompletos. Un hogar de estrato medio-bajo, es aquel cuya jefatura tiene estudios primarios completos o secundarios incompletos. Un hogar de estrato bajo, es aquel cuya jefatura no tiene estudios o tiene estudios primarios incompletos.

${ }^{7}$ Relaciona a la población de setenta y cinco años o más con la población de 65 años o más. Indicador que registra el fenómeno del envejecimiento del envejecimiento.

${ }^{8}$ Relaciona a la población menor de quince años con la población mayor de sesenta y cuatro años.

${ }^{9}$ Relaciona a la población mayor de sesenta y cuatro años con la población menor de quince años.

${ }^{10} \mathrm{Al}$ compartir lo reseñado por diversos especialistas en este campo, los resultados del análisis factorial se ven afectados por decisiones discrecionales adoptadas por el investigador, a saber: modo de factorizar, tipo de rotación utilizada, factores seleccionados, entre los más relevantes. En esta línea argumental, resulta no solo honesto, sino que necesario, en términos de resguardar el rigor de este examen, explicitar las decisiones adoptadas a este respecto.

${ }^{11}$ Entre los investigadores que se han abocado al examen metodológico de este tipo de técnicas de análisis multivariable (Lavia, 1995; RodríguezJaume, 2000), existe coincidencia en que esta exigencia solo es imperativa cuando se trabaja con una muestra y no lo es cuando se trabaja con el universo, como es el caso de este estudio en donde se examina a todo el universo poblacional del área metropolitana de Valparaíso.
} 
Tabla 4

Resultados Test de Linealidad 1992, 2002 y 2017.

\begin{tabular}{llll}
\hline Test & Significación 1992 & Significación 2002 & Significación 2017 \\
\hline Determinante & $5,401 \mathrm{E}-37$ & $3,447 \mathrm{E}-28$ & $1,816 \mathrm{E}-25$ \\
\hline Test de Esfericidad de Bartlett & 0,000 & 0,000 & 0,000 \\
\hline Índice KMO de Káiser-Meyer-Olkin & 0,670 & 0,804 & 0,790 \\
\hline
\end{tabular}

Fuente: Elaboración propia.

factorial, son el determinante, el de esfericidad de Bartlett y el índice KMO de Káiser-Meyer-Olkin (Tabla 4).

Al acreditarse la intercorrelación entre los indicadores de las respectivas matrices, se procedió al análisis factorial. La opción de análisis factorial adoptada fue la de componentes principales. Esta decisión se sustentó en la necesidad del estudio de utilizar los factores resultantes en los procesos analíticos y agrupamientos espaciales posteriores ${ }^{12}$.

En relación al número de factores a obtener en las soluciones factoriales de cada año, la decisión fue no delimitar previamente este número utilizando la regla de Kaiser que pone como condición, para considerar un factor en el análisis, el que sus valores propios sean superiores a "1". Según este protocolo, la estructura factorial obtenida consideró cuatro factores, en los tres años examinados, 1992, 2002 y 2017, que explican en conjunto el 88,6\%, el $87,9 \%$ y el $85,3 \%$ de la varianza registrada en las respectivas matrices, lo que califica como soluciones factoriales apropiadas (Tabla 5).

El contenido de los factores, es decir qué indicadores sintetiza, viene dado por los pesos factoriales de cada indicador en el factor. Como una forma de facilitar la interpretación del contenido de los factores relevados en el análisis, se optó por realizar una rotación factorial obteniendo una nueva matriz a través de la aplicación de la rotación Varimax ${ }^{13}$ (Tabla 5).

Además de considerar en la matriz factorial los valores propios o eigenvalues 14 de cada variable, se consideró necesario, como sugieren los expertos metodólogos, también distinguir las comunalidades 15 de los indicadores (Tablas 6 y 7).

Tabla 5

Estructura factorial reducida por Análisis de Componentes Principales.

\begin{tabular}{|c|c|c|c|c|c|c|c|c|c|}
\hline \multirow[b]{2}{*}{ Factor } & \multicolumn{3}{|c|}{ Estructura Factorial Año 1992} & \multicolumn{3}{|c|}{ Estructura Factorial Año 2002} & \multicolumn{3}{|c|}{ Estructura Factorial Año 2017} \\
\hline & Valor Propio & $\begin{array}{l}\text { \% Varianza } \\
\text { Explicad }\end{array}$ & $\begin{array}{l}\% \text { Varianza } \\
\text { Explicada } \\
\text { Acumulad }\end{array}$ & Valor Propio & $\begin{array}{l}\% \text { Varianza } \\
\text { Explicad }\end{array}$ & $\begin{array}{l}\text { \% Varianza } \\
\text { Explicada } \\
\text { Acumulad }\end{array}$ & Valor Propio & $\begin{array}{l}\% \text { Varianza } \\
\text { Explicad }\end{array}$ & $\begin{array}{l}\text { \% Varianza } \\
\text { Explicada } \\
\text { Acumulad }\end{array}$ \\
\hline 1 & 10,043 & 37,195 & 37,195 & 8,962 & 33,194 & 33,194 & 8,635 & 33,212 & 33,212 \\
\hline 2 & 7,772 & 28,784 & 65,978 & 7,518 & 27,846 & 61,040 & 6,409 & 24,648 & 57,860 \\
\hline 3 & 4,102 & 15,194 & 81,172 & 4,133 & 15,309 & 76,349 & 3,902 & 15,007 & 72,868 \\
\hline 4 & 2,000 & 7,408 & 88,581 & 3,116 & 11,540 & 87,889 & 3,219 & 12,382 & 85,250 \\
\hline
\end{tabular}

Fuente: Elaboración propia.

${ }^{12}$ Las puntuaciones factoriales para cada una de las zonas censales que se obtienen una vez aplicada esta técnica solo es exacta si el método de extracción utilizado es el de componentes principales.

13 "La rotación Varimax sigue criterios de rotación ortogonales según los cuales los factores comunes no están correlacionados. Para facilitar la interpretación factorial minimiza el número de variables que tienen saturaciones altas en un mismo factor y bajas en el resto. Deja invariantes las comunalidades y obtiene una matriz que corresponde también a factores ortogonales (RodríguezPérez, 2004; Rodríguez-Jaume, 2000).

${ }^{14}$ En la matriz solo se consideraron los valores propios con pesos superiores a 0,5 .

${ }^{15}$ La comunalidad mide el grado de representación o participación de cada indicador en la estructura factorial resultante y su valor oscila entre 0 y 1. Cuando se aproxima a 1 indica que el indicador queda totalmente explicado por los factores, mientras que, si se aproxima a 0 , los factores no explican nada de la variabilidad del indicador. Se utiliza para interpretar correctamente la solución factorial ya que no todos los pesos factoriales se asocian a fuertes correlaciones (Rodríguez-Jaume, 2000). 
Tabla 6

Soluciones factoriales rotadas años 1992 y 2002.

\begin{tabular}{|c|c|c|c|c|c|c|c|c|c|c|c|}
\hline \multicolumn{6}{|c|}{ Año 1992} & \multicolumn{6}{|c|}{ Año 2002} \\
\hline Variables & F1 & F2 & F3 & F4 & Comunalidad & Variables & F1 & F2 & F3 & F4 & Comunalidad \\
\hline V19 & 0,733 & & & & 0,762 & $\mathrm{v} 19$ & & 0,695 & & & 0,795 \\
\hline V20 & & 0,904 & & & 0,948 & v20 & & 0,890 & & & 0,903 \\
\hline V14 & & 0,715 & & & 0,842 & v14 & & 0,620 & & 0,625 & 0,910 \\
\hline V15 & 0,925 & & & & 0,982 & v15 & 0,934 & & & & 0,966 \\
\hline V16 & 0,881 & & & & 0,935 & $\mathrm{v} 16$ & 0,895 & & & & 0,935 \\
\hline V21 & 0,941 & & & & 0,976 & v17 & 0,709 & & & & 0,746 \\
\hline V22 & & 0,951 & & & 0,954 & v18 & & & & 0,673 & 0,863 \\
\hline V5 & & 0,866 & & & 0,854 & v23 & 0,809 & & & & 0,810 \\
\hline V23 & 0,861 & & & & 0,890 & v24 & & 0,843 & & & 0,814 \\
\hline V24 & & 0,815 & & & 0,783 & v25 & & & 0,929 & & 0,905 \\
\hline V25 & & & 0,913 & & 0,939 & v27 & & 0,838 & & & 0,770 \\
\hline V27 & & 0,864 & & & 0,750 & v26 & & & 0,938 & & 0,921 \\
\hline V26 & & & 0,855 & & 0,915 & v6 & 0,884 & & & & 0,908 \\
\hline V6 & 0,868 & & & & 0,974 & v7 & 0,750 & & & & 0,926 \\
\hline V7 & 0,803 & & & & 0,863 & v8 & 0,933 & & & & 0,954 \\
\hline V8 & 0,854 & & & & 0,974 & v9 & 0,924 & & & & 0,971 \\
\hline V13 & & 0,699 & & & 0,780 & v13 & & & & 0,779 & 0,880 \\
\hline V12 & & & & $-0,917$ & 0,871 & $\mathrm{v} 12$ & & & 0,665 &,- 585 & 0,806 \\
\hline V11 & & & & 0,693 & 0,585 & v11 & & & & 0,748 & 0,658 \\
\hline V10 & & 0,646 & $-0,651$ & & 0,855 & $\mathrm{v} 1$ & 0,518 & & 0,743 & & 0,930 \\
\hline V1 & 0,536 & & 0,792 & & 0,979 & v2 & 0,706 & & 0,543 & & 0,941 \\
\hline V2 & 0,849 & & & & 0,963 & v3 & 0,924 & & & & 0,888 \\
\hline V3 & 0,924 & & & & 0,960 & v4 & & 0,866 & & & 0,912 \\
\hline V4 & & 0,798 & & & 0,861 & v10 & & 0,713 &,- 536 & & 0,861 \\
\hline V18 & & 0,754 & & & 0,906 & v5 & & 0,899 & & & 0,881 \\
\hline V17 & 0,656 & 0,556 & & & 0,841 & v21 & 0,831 & & & & 0,927 \\
\hline V9 & 0,904 & & & & 0,975 & v22 & & 0,912 & & & 0,948 \\
\hline
\end{tabular}

Fuente: Elaboración propia.

La interpretación de los resultados involucró analizar las saturaciones factoriales significativas de cada factor que considera tanto los valores positivos como negativos y denominar el factor, en un diálogo con las referencias teóricas y empíricas consultadas en la investigación.

\section{Resultados}

En los tres años examinados, los indicadores que informan de las migraciones, intercomunales e internacionales y de corta y larga trayectoria, conforman los factores de mayor peso explicativo en la solución (Tablas 6 y 7). 
Tabla 6

Soluciones factoriales rotadas años 1992 y 2002.

\begin{tabular}{|c|c|c|c|c|c|}
\hline \multicolumn{6}{|c|}{ Año 2017} \\
\hline Variables & F1 & F2 & F3 & F4 & Comunalidades \\
\hline v19 & & 0,754 & & & 0,827 \\
\hline v20 & & 0,873 & & & 0,823 \\
\hline v21 & 0,826 & & & & 0,929 \\
\hline v22 & & 0,897 & & & 0,848 \\
\hline v14 & & 0,669 & & & 0,834 \\
\hline v17 & 0,794 & & & & 0,683 \\
\hline v15 & 0,815 & & & & 0,932 \\
\hline v18 & & 0,531 & & 0,621 & 0,803 \\
\hline v15 & 0,911 & & & & 0,950 \\
\hline v23 & 0,723 & & & & 0,817 \\
\hline v24 & & 0,854 & & & 0,776 \\
\hline v25 & & & 0,866 & & 0,784 \\
\hline v26 & & & 0,858 & & 0,893 \\
\hline v27 & & 0,651 & & & 0,598 \\
\hline v6 & 0,910 & & & & 0,973 \\
\hline v7 & 0,827 & & & & 0,871 \\
\hline v9 & 0,952 & & & & 0,951 \\
\hline v8 & 0,942 & & & & 0,949 \\
\hline v13 & & & & 0,690 & 0,837 \\
\hline v12 & & & & $-0,755$ & 0,773 \\
\hline v11 & & & & 0,820 & 0,763 \\
\hline v5 & & 0,741 & & & 0,733 \\
\hline $\mathrm{v} 1$ & & & 0,730 & & 0,903 \\
\hline v2 & & & 0,658 & & 0,947 \\
\hline v3 & 0,697 & & & & 0,834 \\
\hline v4 & & 0,719 & & & 0,910 \\
\hline v10 & & 0,760 & & & 0,913 \\
\hline
\end{tabular}

Fuente: Elaboración propia.

El año 2017, el primer factor, que explica el 33,2\% de la varianza de la solución factorial, está conformado por indicadores que informan de las zonas urbanas que concentran la migración intercomunal de larga trayectoria (población nacida en otra comuna), de hogares familiares (hogares tipo nuclear, extendido y compuesto), de viviendas particulares tipo casa, de la situación laboral (hombres y mujeres activos e inactivos económicamente) y de los hogares de estrato socioeconómico medio. Los mismos indicadores son sintetizados por el primer factor en las soluciones factoriales correspondiente a los años 1992 y 2002, que suma eso sí indicadores que informan de migraciones intercomunales recientes 16 y de hogares de estrato medio-bajo y bajo, respectivamente. Explican el $37,2 \%$ y el $33,2 \%$ de la varianza total, respectivamente.

El segundo factor que se distingue en la solución factorial del año 2017, que explica el 24,6\% de la varianza, sintetiza indicadores que informan de las zonas urbanas que concentran las migraciones recientes 17 , intercomunales e internacionales, las migraciones internacionales de larga trayectoria18, los hogares tipo no familiar (hogares unipersonal y sin núcleo), las viviendas particulares tipo departamento en edificio y las viviendas desocupadas, los hogares de estrato socioeducativo medio-alto y alto y las jefaturas de hogar con altos niveles de escolaridad (estudios de posgrado y años de estudio promedio). Tanto el año 1992 como el año 2002 el segundo factor, que explica el $28,8 \%$ y $27,8 \%$ de la varianza, respectivamente, sintetiza prácticamente los mismos indicadores que la solución factorial registrada el año 2017, con la salvedad de que incluyen indicadores referidos al envejecimiento poblacional (índice de vejez) y a hogares tipo familiar (hogares compuestos), en el caso del año 1992 y excluye el indicador de hogar sin núcleo, en el caso del año 2002.

El tercer factor de las soluciones factoriales correspondientes a los años 1992, 2002 y 2017, que explican el 15,2\%, el $15,3 \%$ y el $15 \%$ de sus respectivas varianzas, sintetizan indicadores que informan del hábitat de los hogares más desventajados socioeconómicamente (hogares de estrato medio-bajo y bajo) y de viviendas particulares precarias (viviendas tipo no permanente y de calidad global irrecuperable). El año 2002, incorpora además indicadores que informan de zonas de más concentración de población joven (índice de juventud) y de jefaturas de hogar de menor escolaridad (años de estudio promedio).

El cuarto factor, que sintetiza indicadores que refieren al proceso de envejecimiento de la población, característico del proceso de segunda transición demográfica que emergería

\footnotetext{
${ }^{16}$ Se refiere con este constructo a la población residente en Viña del Mar que hace cinco años residía en otra comuna del país

17 Ídem.

${ }^{18}$ Se refiere a la población que nació en otro país.
} 
Tabla 8

Matriz de correlaciones población migrante-tipos de hogar.

\begin{tabular}{l|ccccc}
\hline \multirow{2}{*}{ Población migrante } & \multicolumn{5}{c}{ Tipos de hogar } \\
\cline { 2 - 6 } & Unipersonal & Compuesto & Extendido & Sin Núcleo & Nuclear \\
\hline Reciente interior & 0,714 & 0,388 & $-0,054$ & 0,684 & 0,387 \\
\hline Reciente internacional & 0,695 & 0,224 & $-0,338$ & 0,600 & 0,056 \\
\hline Nacido en otra comuna & 0,612 & 0,677 & 0,416 & 0,578 & 0,778 \\
\hline Nacido en otro país & 0,711 & 0,315 & $-0,276$ & 0,599 & 0,140 \\
\hline
\end{tabular}

Fuente: Elaboración propia.

Tabla 9

Matriz de correlaciones población migrante-hogares estrato socioeducativo.

\begin{tabular}{l|cccc}
\hline \multirow{2}{*}{ Población Migrante } & \multicolumn{4}{c}{ Estrato socioeducativo de los hogares } \\
\cline { 2 - 5 } & Alto & Medio-Alto & Medio-Bajo & Bajo \\
\hline Reciente interior & $-0,261$ & $-0,303$ & $-0,054$ & 0,816 \\
\hline Reciente internacional & $-0,418$ & $-0,473$ & $-0,311$ & 0,682 \\
\hline Nacido en otra comuna & 0,044 & 0,050 & 0,361 & 0,738 \\
\hline Nacido en otro país & $-0,315$ & $-0,384$ & $-0,268$ & 0,701 \\
\hline
\end{tabular}

Fuente: Elaboración propia.

con fuerza en el área metropolitana de Valparaíso, en general, y en Viña del Mar, en particular. En el período en estudio incrementan su participación en la explicación de la varianza de la solución factorial, de un 7,4\% registrado el año 1992 a un $11,5 \%$ y $12,4 \%$ el año 2002 y 2017 , respectivamente. Este último año, sintetiza los indicadores que refieren a las zonas que concentran más hogares sin núcleo y registran una estructura demográfica más envejecida, es decir, más alta proporción de adultos mayores en relación a la población menor de quince años (índice de vejez e índice de juventud) y más alta proporción de población de setenta y cinco años o más en relación a la población de sesenta y cinco años o más (índice de longevidad).

Al observar la matriz de correlaciones, se registran relaciones estadísticamente significativas entre las zonas que concentran a los migrantes recientes, intercomunales e internacionales, con las zonas que concentran los tipos de hogar no familiar (Tabla 8).

La positiva, significativa y alta correlación registrada en los tres años en estudio, entre la concentración espacial de la población migrante y la concentración de hogares de estrato socioeducativo alto en estas zonas residenciales (Tabla 9), es coherente con lo registrado por la Encuesta de Caracterización Socioeconómica Nacional, CASEN, en el año 2015, último año disponible en que se diseñó una muestra estadísticamente representativa del nivel comunal.

Al analizar el perfil socioeconómico del residente reciente ${ }^{19}$, observamos que el $44 \%$ se concentra en el quintil de más altos ingreso autónomo nacional (Tabla 10).

También es significativa, para los fines de este estudio, la alta y significativa correlación observada entre las zonas que concentran más población inmigrante internacional, de larga o reciente trayectoria, con las zonas que concentran más stock de viviendas particulares tipo departamentos y desocupadas (Tabla 11). Ello da cuenta de la significativa participación del mercado inmobiliario habitacional en la atracción y distribución segregada, socioeconómica y territorialmente, de la migración intercomunal e internacional, como lo hemos referido en estudios anteriores (Valdebenito, 2017).

En el espacio residencial, se registra una huella nítida de la relación estadísticamente acreditada en el examen factorial de componentes principales, entre la distribución de los migrantes de larga y corta trayectoria, interior e internacional, con la distribución territorial de los hogares de estrato socioeducativo alto y de las viviendas

\footnotetext{
${ }^{19}$ De acuerdo a la encuesta CASEN, refiere a la población residente en Viña del Mar el año 2015, que el año 2010 residía en otra comuna o país.
} 
Tabla 10

Población migrante reciente, según decil de ingreso autónomo nacional.

\begin{tabular}{|c|c|c|c|c|c|c|c|c|c|c|c|}
\hline \multirow{2}{*}{ Comunas } & \multicolumn{10}{|c|}{ Porcentaje de población según decil de ingreso autónomo nacional } & \multirow{2}{*}{ Total } \\
\hline & I & II & III & IV & V & VI & VII & VIII & IX & $\mathbf{x}$ & \\
\hline Viña del Mar & 3,9 & 9,0 & 3,0 & 2,8 & 10,7 & 6,0 & 10,2 & 10,4 & 20,4 & 23,6 & 100,0 \\
\hline Gran Valparaíso & 4,2 & 9,1 & 8,0 & 6,5 & 13,0 & 6,8 & 10,4 & 11,2 & 16,6 & 14,1 & 100,0 \\
\hline
\end{tabular}

Fuente: Elaboración propia. Encuesta CASEN 2015.

Tabla 11

Matriz de correlaciones población migrante-tipos de viviendas particulares.

\begin{tabular}{l|ccccc}
\hline \multirow{2}{*}{ Población migrante } & \multicolumn{5}{c}{ Tipos de viviendas particulares } \\
\cline { 2 - 6 } & Casa & Departamentos & No permanentes & Irrecuperables & Desocupadas \\
\hline Reciente interior & $-0,106$ & 0,607 & $-0,124$ & $-0,210$ & 0,208 \\
\hline Reciente internacional & $-0,337$ & 0,714 & $-0,237$ & $-0,359$ & 0,466 \\
\hline Nacido en otra comuna & 0,313 & 0,343 & 0,015 & 0,020 & $-0,055$ \\
\hline Nacido en otro país & $-0,247$ & 0,680 & $-0,108$ & $-0,235$ & 0,460 \\
\hline
\end{tabular}

Fuente: Elaboración propia.

particulares desocupadas, destinadas a arriendo o venta. Las zonas residenciales emplazadas en el plan y en el borde costero de Viña del Mar destacan por concentrar, a la vez, los porcentajes más altos de viviendas particulares desocupadas (Figura 2), de hogares de más alto estatus social (Figura 3), de migración reciente interior (Figura 4) e internacional (Figura 5) y de nacidos en otra comuna (Figura 6) u otro país (Figura 7).

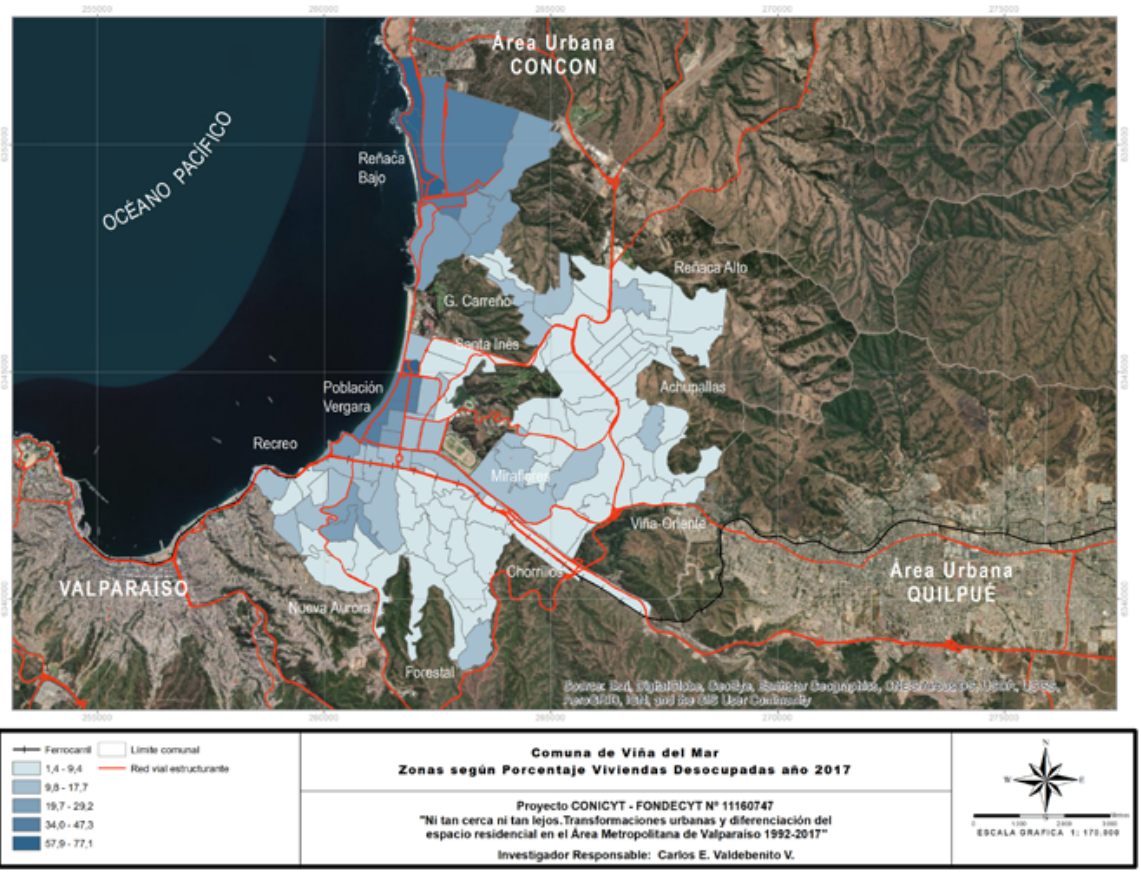

Figura 2. Zonas residenciales según porcentaje de viviendas desocupadas, año 2017. Fuente: Elaboración propia. 

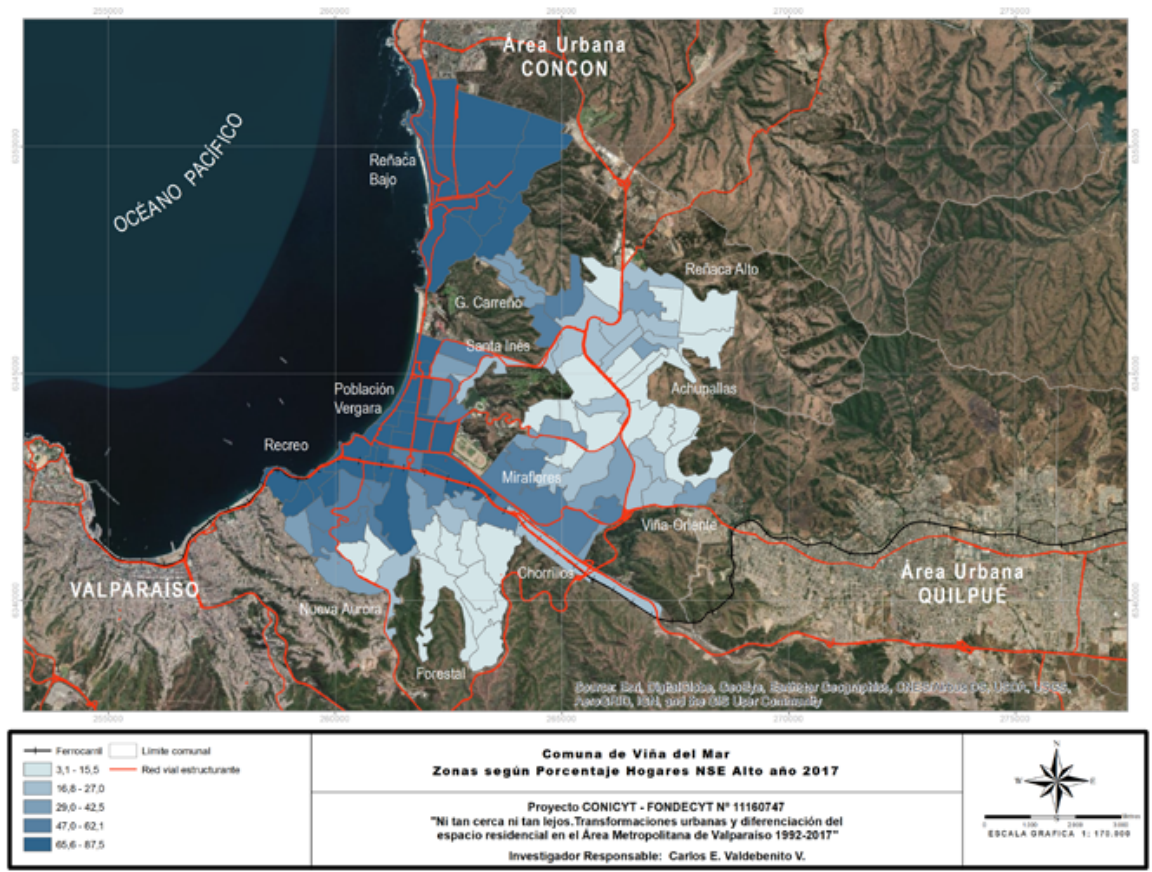

Figura 3. Zonas residenciales según porcentaje de hogares de estrato socioeducativo alto, año 2017. Fuente: Elaboración propia.
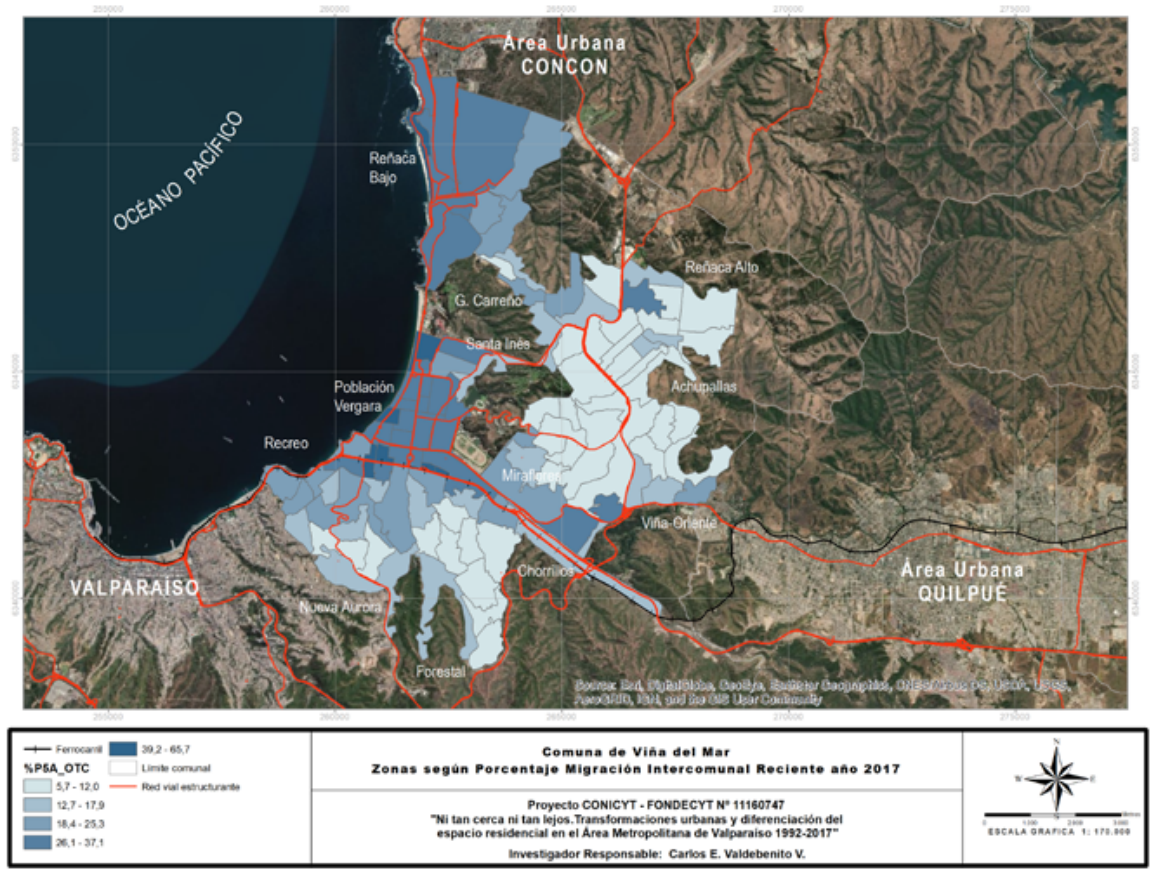

Figura 4. Zonas residenciales según porcentaje de migración reciente interior, año 2017. Fuente: Elaboración propia. 

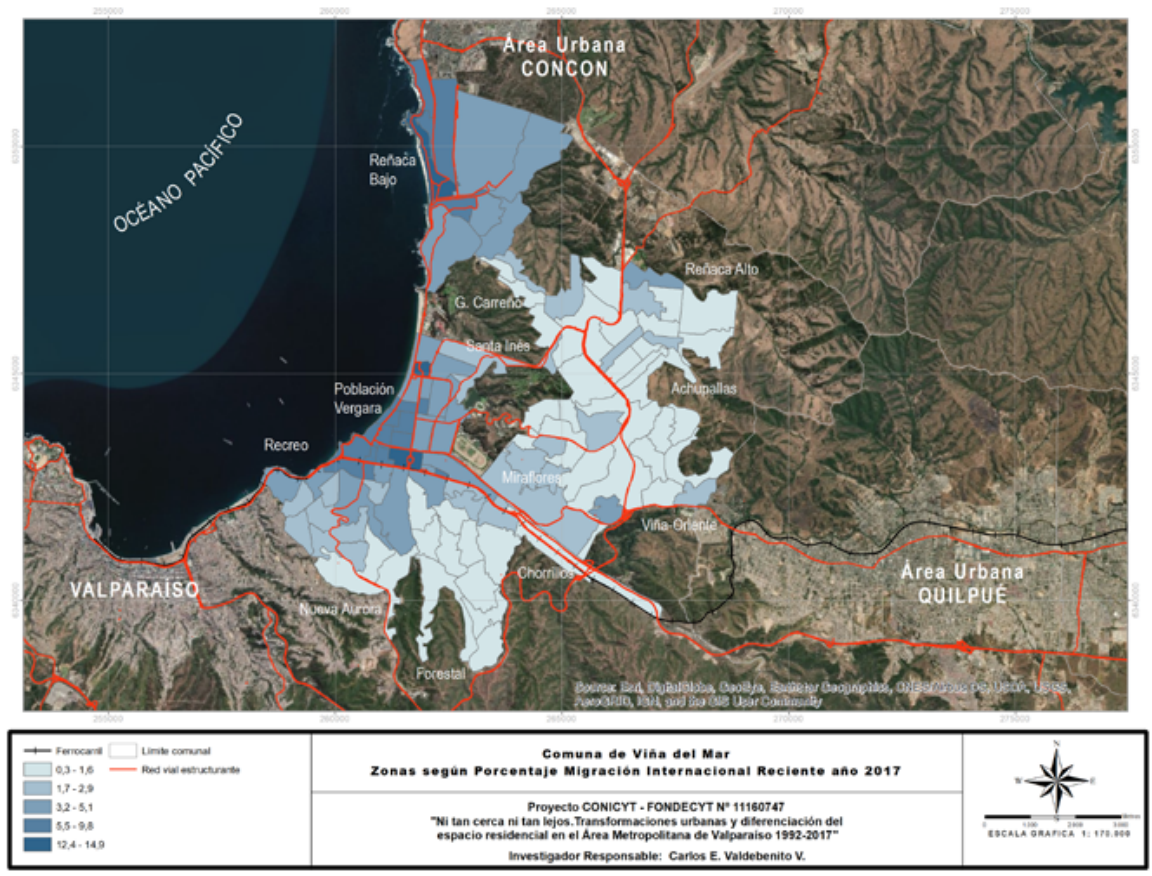

Figura 5. Zonas residenciales según porcentaje de migración reciente internacional, año 2017. Fuente: Elaboración propia.
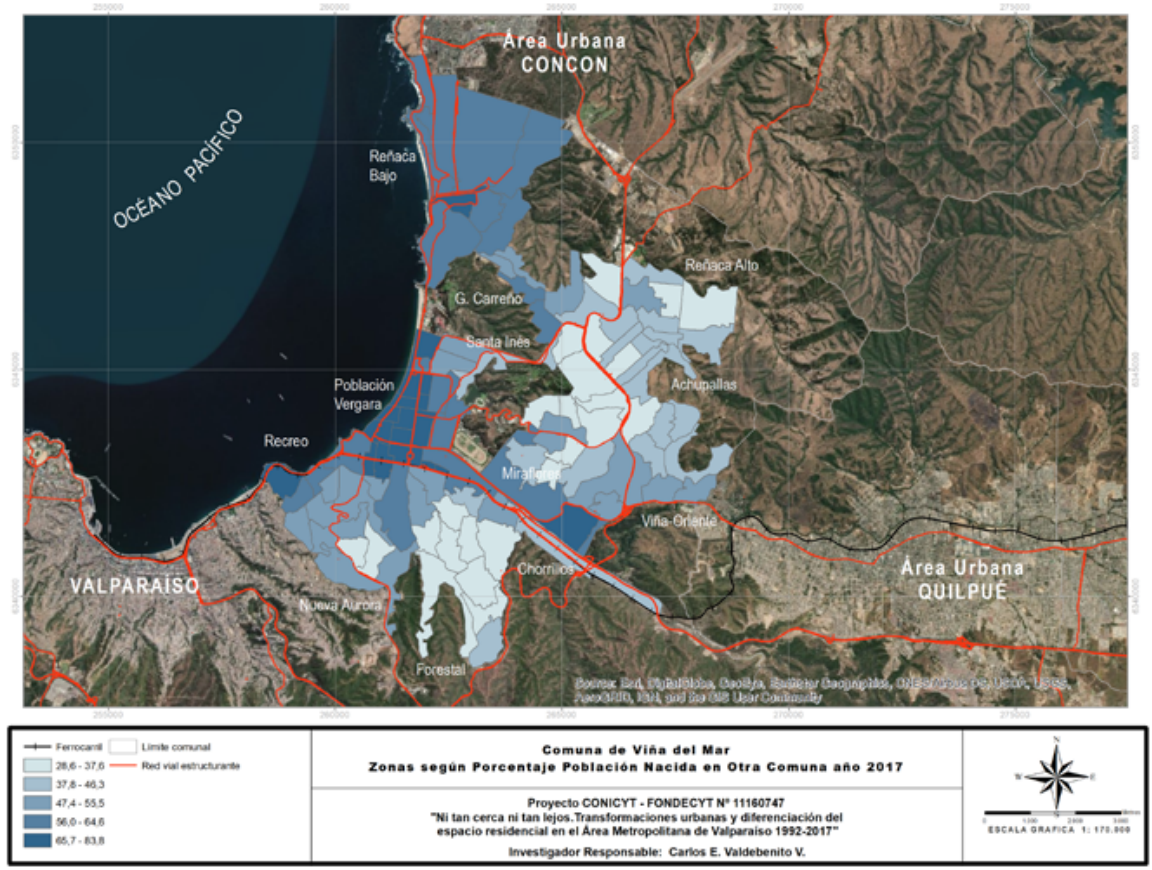

Figura 6. Zonas residenciales según porcentaje de población nacida en otra comuna, año 2017. Fuente: Elaboración propia. 


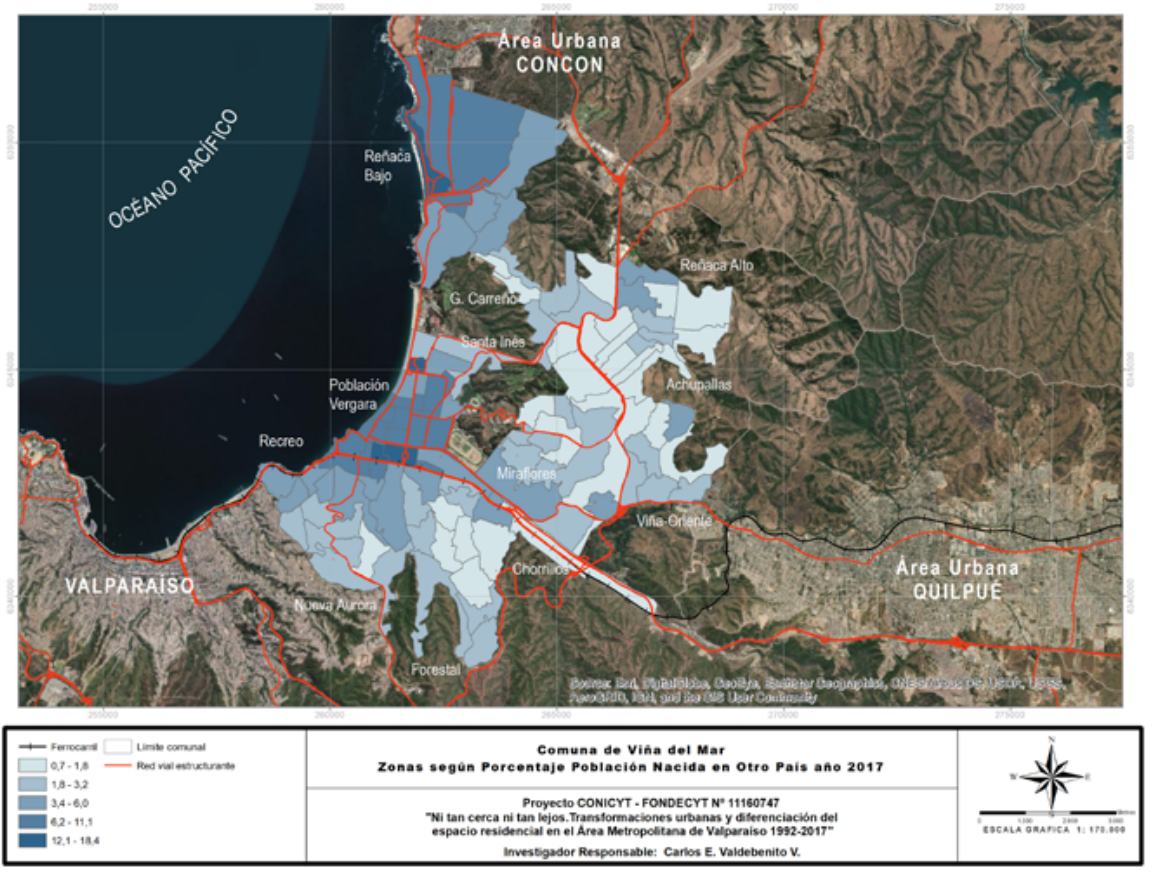

Figura 7. Zonas residenciales según porcentaje de población nacida en otro país, año 2017. Fuente: Elaboración propia.

\section{Conclusiones}

El patrón sociodemográfico que se distingue en las soluciones factoriales que resultan del análisis realizado a Viña del Mar, tanto para el año 2017 como para los años 2002 y 1992, matiza el factor socioeconómico predominante en la estructura socioresidencial de las ciudades latinoamericanas.

Hay zonas residenciales diferenciadas en la ciudad, no solo en términos socioeconómicos sino también sociodemográficos, las que se han consolidado en su configuración segregada por los procesos migratorios, especialmente los de carácter intercomunal dado su volumen. Los migrantes en función de su estrato socioeconómico, primero, y su perfil sociodemográfico, después, tienen zonas residenciales en las que es más probable que pueda o no pueda establecerse. El papel de los agentes urbanos privados del negocio inmobiliario en este proceso de segregación ha sido protagónico, aunque no exclusivo.

La movilidad residencial intercomunal, que tiene como destino el área metropolitana de Valparaíso en general y la ciudad de Viña del Mar en particular, ha sido facilitada por diversos actuaciones públicas, normativas e infraestructurales que han traído como correlato no solo el mejoramiento de la conectividad intercomunal e interregional, sino también, y sobre todo, la generación de suelo urbano y de nuevas condiciones de uso de estos que han favorecido a los agentes privados del negocio inmobiliario. Entre estas actuaciones en la ciudad de Viña del Mar, de carácter infraestructural, destacan la modernización y soterramiento de la red técnica ferroviaria que conecta a las ciudades de Valparaíso, Viña del Mar, Quilpué, Villa Alemana y Limache, así como la reposición y construcción, bajo la modalidad de concesión, de redes viales de alto estándar de conexión intracomunal, intercomunal e interregional.

A contramano de lo evidenciado en ciudades metropolitanas latinoamericanas, el suelo urbano que se ha construido en este último período intercensal, para acoger a los nuevos residentes, no ha traído como correlato, ni evidente ni recurrente, procesos de gentrificación y desplazamiento residencial. Si bien hay actuaciones en la ciudad que pueden inscribirse en este fenómeno, lo que ha predominado en la generación de nuevo suelo urbano, es la ocupación de espacios que habían quedado como remanentes al interior de la ciudad consolidada y la ocupación de espacios intersticiales en la conurbación costera Viña del Mar-Concón (que podrían caber en el fenómeno de la acumulación por desposesión, dado los capitales ambientales que se han hipotecado en favor de las actuaciones inmobiliarias privadas). 
No hay duda que el mejoramiento y construcción de redes técnicas de transporte, vial y ferroviaria, han contribuido creciente y significativamente a la integración de la ciudad de Viña del Mar con el área metropolitana de Valparaíso que conforma, sin embargo, a partir de los resultados obtenidos en este examen, observamos una aparente paradoja, la integración espacial que posibilita, amplía complementariamente el campo de probabilidades para la segregación residencial de tipo socioeconómica, más aún si consideramos que son decisiones no solo privadas, sino principalmente públicas, las que han favorecido el negocio especulativo inmobiliario, lo que vuelve a materializar la segregación residencial de tipo socioeconómica de gran escala, tan propia de nuestras metrópolis latinoamericanas. A los pobres o los más desfavorecidos socioeconómicamente que, aunque reducidos en tamaño, también llegan a la ciudad, dado lo segregado de la oferta inmobiliaria formal, en favor de una demanda de más alto estatus socioeconómico, solo les queda como camino el engrosar el tamaño de la población que reside informalmente en la encumbrada y menos conectada periferia de la ciudad.

\section{Referencias}

Contreras, Y. (2017). De los "gentries" a los precarios urbanos. Los nuevos residentes del centro del Santiago. Revista EURE (Santiago), 43(129). https:// doi.org/10.4067/S0250-71612017000200006

Contreras Gatica, Y., Ala-Louko, V. \& Labbé, G. (2015). Acceso exclusionario y racista a la vivienda formal e informal en las áreas centrales de Santiago e Iquique. Polis (Santiago), 14(42), 53-78. https:// dx.doi.org/10.4067/S0718-65682015000300004

Checa, J. C. \& Arjona, A. (2006). Ecología factorial en Roquetas de Mar (Almería). La importancia de las migraciones en la configuración socioespacial. Scripta Nova, Revista Electrónica de Geografía y Ciencias Sociales, 10(219).

Duhau, E. (2013). La división social del espacio metropolitano. Una propuesta de análisis. Nueva Sociedad, 243, 79-91.

Escolano Utrilla, S., Ortiz Véliz, J. \& Moreno Mora, R. (2018). Dinámica y estructura de las migraciones residenciales interurbanas en Chile, 19872002. Revista INVI, 33(94), 105-133. https://doi. org/10.4067/S0718-83582018000300105
Florida, R. (2017). La nueva crisis urbana: cómo nuestras ciudades aumentan la desigualdad, profundizan la segregación y fallan a la clase media, y qué podemos hacer al respecto. Nueva York: Libros básicos.

Gil-Alonso,F.;Bayona,J.\&Pujadas,I.(2013).Fromboom to crash: Spanish urban areas in a decade of change (2001-2011). European Urban and Regional Studies, 3(2). https://doi.org/10.1177/0969776413498762

Gil-Alonso, F., Bayona, J. \& Pujadas, I. (2015). Las migraciones internas de los extranjeros en España: Dinámicas espaciales recientes bajo el impacto de la crisis. Boletín de la Asociación de Geógrafos Españoles, 69, 233-261. https://doi.org/10.21138/ bage. 1896

González, S. \& Villeneuve, P. (2006). Transformaciones en el espacio socioresidencial de Monterrey, 19902000. Estudios Demográficos y Urbanos, 22(1), 143178. https://doi.org/10.24201/edu.v22i1.1296

Link, F., Valenzuela, F. \& Fuentes, L. (2015). Segregación, estructura y composición social del territorio metropolitano en Santiago de Chile: Complejidades metodológicas en el análisis de la diferenciación social en el espacio. Revista de geografia Norte Grande, 62, 151-168. https://dx.doi. org/10.4067/S0718-34022015000300009

INE - DEM [Instituto Nacional de Estadísticas Departamento de Extranjería y Migración]. (2019). Estimación de personas extranjeras residentes en Chile al 31 de diciembre 2018. Informe Metodológico. Santiago de Chile: Instituto Nacional de Estadísticas y Departamento de Extranjería y Migración.

INE [Instituto Nacional de Estadísticas] (2018). Estimaciones y proyecciones de la población de Chile 1992-2050. Total país. Metodología y principales resultados. Santiago de Chile: Instituto Nacional de Estadísticas.

Janoschka, M. (2016). Gentrificación, desplazamiento, desposesión: procesos urbanos claves en América Latina. Revista INVI, 31(88), 27-71. https://doi. org/10.4067/S0718-83582016000300002

Leal, J. \& Sorando, D. (2016). Economic crisis, social change and segregation processes in Madrid. 
En Tammaru, T. \& S. Musterd. Socio-economic segregation in European capital cities. Londres: Routledge.

Marcos, M. \& Mera, G. (2018). Migración, vivienda y desigualdades urbanas: condiciones sociohabitacionales de los migrantes regionales en Buenos Aires. Revista INVI, 33(92), 53-86. https:// doi.org/10.4067/S0718-83582018000100053

Matossian, B. (2015). División social del espacio residencial y migraciones: El caso de San Carlos de Bariloche, Argentina. EURE (Santiago), 41(124), 163-184. https://dx.doi.org/10.4067/S025071612015000400008

Matossian, B. (2010). Expansión urbana y migración. El caso de los migrantes chilenos en San Carlos de Bariloche como actores destacados en la conformación de barrios populares. Scripta Nova. Revista Electrónica de Geografía y Ciencias Sociales, 14(331).

Musterd, S., Marcińczak,S., Van Ham, M. \& Tammaru, T. (2017). Socioeconomic segregation in European capital cities. Increasing separation between poor and rich. Urban Geography, 38(7), 1062-1083. https:// doi.org/10.1080/02723638.2016.1228371

Nel-lo,O. (2015). La ciudad en movimiento: Crisis social y respuesta ciudadana. Madrid: Díaz \& Pons.

Ortiz, J. \& Schiappacasse, P. (1997). Las migraciones intraurbanas y su impacto en la diferenciación del espacio social del Gran Santiago. Revista Geográfica de Chile Terra Australis, 42, 121-138. Recuperado de https://scielo.conicyt.cl/scielo.php?script=sci arttext\&pid=S0250-71612002008500009

Ortiz, JoJ. \& Morales, S. (2002). Impacto socioespacial de las migraciones intraurbanas en entidades de centro y de nuevas periferias del Gran Santiago. EURE (Santiago), 28(85), 171-185. https://dx.doi. org/10.4067/S0250-71612002008500009

Ortiz, J. \& Escolano, S. (2013). Movilidad residencial del sector de renta alta del Gran Santiago (Chile): hacia el aumento de la complejidad de los patrones socioespaciales de segregación. EURE (Santiago), 39(118), 77-96. https://dx.doi. org/10.4067/S0250-71612013000300004
Pujadas-i-Rúbies, I. (2009). Movilidad residencial y expansión urbana en la Región Metropolitana de Barcelona, 1982-2005. Scripta Nova: Revista Electrónica de Geografía y Ciencias Sociales, 13(290). Recuperado de http://www.ub.edu/geocrit/ sn/sn-290.htm

Rodríguez-Jaume, M. (2000). Modelos sociodemográficos: Atlas Social de la Ciudad de Alicante. Tesis para optar al grado de Doctor en Sociología. Biblioteca Virtual Miguel de Cervantes, Universidad de Alicante.

Tammarú, T., Van Ham, M., Marcińczak, S. \& Musterd, S. (2015). A multi-factor approach to understanding socio-economic segregation in European capital cities. En T. Tammarú et al. (eds.). Socio-economic segregation in European capital cities. Londres: Routledge.

Valdebenito, C. (2014). La huella socioeconómica y demográfica en la estructura residencial de las ciudades Revista Gmedias de Latinoamérica: el caso de Viña del Mar-Chile en la década 1992-2002. Scripta Nova. Revista Electrónica de Geografía y Ciencias Sociales, 18(492).

Valdebenito, C. (2017). Ni tan cerca ni tan lejos. Trayectoria de la diferenciación social del espacio residencial en la ciudad de Viña del Mar-Chile. Revista Geográfica de Valparaíso, 54, 1-20.

Zambon, I., Serra, P., Sauri, D., Carlucci, M. \& Salvati, L. (2017). Más allá de la 'ciudad mediterránea': disparidades socioeconómicas y expansión urbana en tres ciudades del sur de Europa. Geografiska Annaler: Serie B, Geografía humana, 99(3), 319- 337. https:// doi.org/10.1080/04353684.2017.1294857 